\title{
TOXICIDADE COMPARATIVA DE LAMBDA- CYALOTHRIN À LAGARTA-DA-SOJA, Anticarsia gemmatalis HUEB., 1818 (LEPIDOPTERA, NOCTUIDAE) E AO PERCEVEJO VERDE, Nezara viridula (L., 1758) (HEMIPTERA, PENTATOMIDAE) ${ }^{1}$
}

\author{
G.C. de BAPTISTA²; J.R.P. PARRA ${ }^{2}$; M. de L. HADDAī ${ }^{2}$ \\ ${ }^{2}$ Departamento de Entomologia-ESALQ/USP, C.P. 9 - CEP: 13418-900 - Piracicaba,SP
}

\begin{abstract}
RESUMO: O objetivo deste estudo foi avaliar, atraves dos valores de dose letal (DL $\left.\mathrm{B}_{0}\right)$, a toxicidade comparativa do inseticida piretróide lambda-cyalothrin a lagarta-da-soja, Anticarsia gemmatalis e a ninfas do percevejo verde, $N$ Nara viridula, tomando-se como padrão de comparaçāoo inseticida organofosforadomonocrotofos. Os experimentos foram realizados em condiçōes de laboratório por técnica de aplicação tópica, sendo usadas lagartas e ninfas, ambas de $3^{\circ}$ instar. As avaliaçōes das mortalidades foram feitas 3, 6, 24, 48 e 72 horas apos o tratamento. $O$ parâmetro $\mathrm{DL}_{\mathrm{so}}$ foi determinado por analises de probit através de software apropriado. Lambda-cyalothrin agiu mais rapidamente do que monocrotofós contra as lagartas, sendo cerca de 140 a 190 vezes mais tóxico para elas e de 20 a 40 vezes mais ativo contra as ninfas. $O$ inseticida piretróde foi ainda de 5 a 9 vezes mais tóxico a lagarta-da-soja do que ao percevejo verde.
\end{abstract}

Descritores: toxicidade comparativa, lambda-cyalothrin, monocrotofós, Anticarsia gemmatalis, Nezara viridula, técnica de aplicaçāo tópica

\section{COMPARATTVE TOXICITY OF LAMBDA-CYHALOTHRIN TO THE VELVETBEAN CATERPILLAR, Anticarsia gemmatalis HUEB., 1818 (LEPIDOPTERA, NOCTUIDAE) AND TO THE SOUTHERN GREEN STINK BUG, Nezara viridula (L., 1758) (HEMIPTERA, PENTATOMIDAE)}

\begin{abstract}
ARSTRACT: The objective of this study was to evaluate the comparative toxicity of the pyrethroid insecticide lambda-cyhalothrin to the velvetbean caterpillar, Anticarsia gemmatalis and to the nymphs of the southern green stink bug, Nezara viridula. The lethal dose $\left(\mathbf{L D}_{50}\right)$ values were compared with the ones of the organophosphorus insecticide monocrotophos. The experiments were performed under laboratory conditions by topical application technique to $3^{\text {rd }}$ instars of both caterpillars and nymphs. Mortality evaluations were made at 3, 6, 24, 48 and 72 hours after treatment. The $\mathrm{LD}_{\mathbf{s e}}$ parameters were determined by probit analyses through appropriate software. Lambda-cyhalothrin acted more rapidly than monocrotophos against the caterpillars, being approximately 140-190 times more toxic than the latter. Lambda-cyhalothrin was 20-40 times more active against the nymphs than monocrotophos. The pyrethroid insecticide was about 5-9 times more toxic to the velvetbean caterpillar than to the southern green stink bug.

Key Words: comparative toxicity, lambda-cyhalothrin, monocrotophos, Anticarsia gemmatalis, Nezara viridula, topical application technique
\end{abstract}

\section{INTRODUÇÃO}

Os danos causados por pragas agrícolas, insetos e ácaros, podem ser de grande intensidade, razão pela qual são empregadas medidas de controle entre as quais as mais importantes são os métodos culturais, físicos, comportamentais, controle biológico e químico. Até há poucas décadas o homem utilizava quase que exclusivamente métodos químicos.

A importância crescente da questão ambiental, em grande parte levantada na obra

\footnotetext{
${ }^{1}$ Pesquisa financiada pela ICI Brasil S.A.

Trabalho vencedor do Concurso $1^{\circ}$ Prêmio Karatê Ciência.
}

Sci. agric., Piracicaba, 52(1):183-188,jan./abr. 1995 
"Primavera Silenciosa", de Rachel Carson, publicada em 1962, provocou dois direcionamentos neste cenário: o primeiro foi a adoção de novo conceito de controle de insetos, chamado de Manejo Integrado de Pragas, (MIP); o segundo foi a busca de novos inseticidas substitutos aos até então existentes (basicamente clorados, organofosforados e carbamatos), menos agressivos ao meio ambiente, aos animais e ao próprio homem. Assim, surgiram os piretróides sintéticos, baseados nas antigas piretrinas naturais, extraídas das flores de crisântemo, como alethrin, permethrin, deltamethrin, bifenthrin, lambdacyalothrin, etc, de usos bastante variados na agricultura, pecuária e saúde pública. $O$ ingrediente ativo lambda-cyalothrin é um dos mais recentes e tem se mostrado de uso versátil, para controle de inúmeras pragas agrícolas, tais como tripes, pulgões, percevejos, lagartas, besouros, moscasbranca, pragas dos cereais, no campo e no armazenamento, além do controle de insetos vetores de doenças de plantas.

Dentre as culturas mais atacadas por pragas no Brasil, destaca-se a da soja, a qual, por essa razão, é a que mais consome inseticidas, cujo mercado foi de US\$ 74 milhões em 1990 e de US\$ 55 milhões em 1991 (ANDEF, 1992)'. Um inseticida usado como referência nessa lavoura é o organofosforado monocrotofós.

Duas das mais importantes pragas da cultura no Brasil são a lagarta-da-soja, Anticarsia gemmatalis Hueb., 1918 (Lepidoptera, Noctuidae) e os percevejos e, dentre estes, Nezara viridula (L. 1758) (Hemiptera, Pentatomidae), que podem causar perdas consideráveis na produção, se não forem controlados (GALLO et al., 1988).

Diversos estudos têm mostrado a possibilidade de utilização de lambda-cyalothrin para controle de várias pragas desta e de outras culturas, como por exemplo para o percevejo verde (MARTINS et al., 1990), ao bicudo-do-algodoeiro (GABRIEL et al., 1990), à lagarta-rosada-doalgodoeiro (MARTINS et al., 1988), etc.

Outros estudos foram feitos acerca da toxicidade e resistência de pragas agrícolas a lambda-cyalothrin, em experimentos de laboratório e de campo, através da determinação de certos parâmetros toxicológicos, tal como a $\mathrm{DL}_{50}$. Em

\footnotetext{
1 Venda de defensivos agrícolas por destinação 1990 $x$ 1991. (Comunicação Pessoal). Ner manuscrito original).
}

Sci. agric., Piracicaba, 52(1):183-188,jan./abr. 1995 alguns deles foi observado que o inseticida apresentava maior toxicidade e menor "spectrum" de resistência, quando comparado com outros piretróides, como para Pseudoplusia includens (LEONARD et al., 1990), Chalcodermes aeneus (N'GUESSAN \& CHALFANT, 1990), Heliothis virescens (ELZEN et al., 1990; LEONARD et al., 1988), Heliothis armigera (SAXENA et al., 1989), além das espécies de cupins, Coptotermes formosanus e Reticulitermes flavipes (SU \& SCHEFFRAHN, 1990).

O objetivo deste estudo foi avaliar, em laboratório, a toxicidade comparativa do inseticida piretróide lambda-cyalothrin à lagarta-da-soja, Anticarsia gemmatalis e ao percevejo verde, Nezara viridula, através dos valores de $\mathrm{DL}_{50}$, tomando-se como padrão de comparação o inseticida monocrotofós.

\section{MATERIAL E MÉTODOS}

No período de maio a agosto de 1991 foram conduzidos quatro ensaios por técnica de aplicação tópica com os inseticidas lambdacyalothrin e monocrotofós: dois usando-se lagartas de $A$. gemmatalis e outros dois com ninfas de $N$. viridula.

1. Bio-ensaios com Anticarsia gemmatalis: Foram realizados os testes com os dois inseticidas e com insetos provenientes de criação própria do Departamento de Entomologia da ESALQ/USP, em Piracicaba - SP. Os testes foram efetuados com lagartas de $3^{\circ}$ ínstar não anestesiadas, sendo utilizados os seguintes produtos técnicos com as respectivas purezas: lambda-cyalothrin $84 \%$ e monocrotofós $80 \%$.

Como trabalho preliminar foram conduzidos testes, visando estabelecer intervalos de doses dos inseticidas capazes de produzirem mortalidades crescentes nos insetos. Para tanto, foram preparadas soluções dos inseticidas em acetona, em concentrações conhecidas, partindo-se de soluções estoques de concentrações de $10 \mathrm{mg}$ do ingrediente ativo por $\mathrm{ml}$ do solvente. Baseando-se nos resultados preliminares, estabeleceram-se pelo menos sete soluções de concentrações conhecidas capazes de produzirem mortalidades entre 0 e $100 \%$, em função de doses crescentes dos tóxicos.

Os insetos da idade estabelecida foram fornecidos em tubos com dieta de GREENE et al. (1976), contendo, cada um cinco lagartas, 
adotando-se a metodologia descrita por PARRA (1986). A aplicação dos inseticidas foi realizada por meio de um micro-aplicador automático Burkard, provido de seringa de $1 \mathrm{ml}$ de capacidade, permitindo a aplicação, na parte dorsal da lagarta, de um volume exato de $1 \mu l$ de solução inseticida. Após o tratamento cada lagarta era recolocada no mesmo tubo de dieta do qual proviera.

Para cada concentração de inseticida foram usados 100 insetos, separados em quatro repetições de 25 indivíduos cada uma. Além dos tratamentos inseticidas foram, também, mantidos lotes testemu-nhas nos quais as lagartas receberam, apenas, $1 \mu \mathrm{l}$ de acetona, em igual número de repetições e de insetos. As mortalidades foram avaliadas após 3, 6, 24, 48 e 72 horas decorridas do tratamento. Para tanto, os insetos eram retirados do interior dos tubos e avaliados quanto a sua habilidade de se locomoverem, mesmo instados com auxílio de um estilete, sendo considerados mortos aqueles que permaneciam imóveis e os altamente intoxicados, assim julgados pela inabilidade de locomoção normal. Os dados foram analisados por via computacional, utilizando-se o software Probit, desenvolvido pelo Setor de Processamento de Dados do Departamento de Entomologia da ESALQ/USP. Foram, assim, obtidos os valores de $\mathrm{DL}_{50} \mathrm{em} \mu \mathrm{g}$ /inseto com seus respectivos IC $\mathrm{C}_{95}$. A fim de se expressar a toxicidade, também avaliada pela $\mathrm{DL}_{50} \mathrm{em} \mu \mathrm{g} / \mathrm{g}$ de inseto, foram tomados pesos individuais de dez lagartas, igualmente de $3^{\circ}$ ínstar, sendo obtido o peso médio unitário de $64 \pm 8 \mathrm{mg}$.
2. Bio-ensaios com Nezara viridula: Foram realizados os testes com os dois inseticidas e com insetos, também, provenientes de criação do Departamento de Entomologia da ESALQ/USP, mantida segundo técnica de CÔRREA-FERREIRA (1985). Os testes foram efetuados com ninfas de $3^{\circ}$ ínstar não anestesiadas do inseto. Os produtos técnicos, os testes preliminares, a técnica de aplicação tópica foram semelhantes aos relatados anteriormente. Após o tratamento, os insetos eram transferidos para uma caixa de papelão cilíndrica com tampa telada, na qual eram colocados grãos de amendoim como fonte de alimentação das ninfas.

Para cada concentração do inseticida foram usados 40 insetos, separados em quatro repetições com dez indivíduos cada uma. Além dos tratamentos com inseticidas foram, também, mantidos lotes testemunhas, nos quais as ninfas receberam, apenas, aplicação de $1 \mu l$ de acetona, em igual número de repetições e de insetos.

As avaliações das mortalidades, $o$ critério para vivo/morto e a análise dos dados foram os mesmos citados para os bio-ensaios anteriores. Foram, assim, obtidos os valores $\mathrm{DL}_{50} \mathrm{em}$ $\mu \mathrm{g} /$ inseto com seus respectivos $\mathrm{IC}_{95}$. A fim de se expressar a toxicidade, também avaliada pela $\mathrm{DL}_{50}$ em $\mu \mathrm{g} / \mathrm{g}$ de inseto, foram tomados os pesos individuais de dez ninfas, igualmente de $3^{\circ}$ ínstar, sendo obtido o peso médio unitário de $2,8 \pm 0,2 \mathrm{mg}$.

\section{RESULTADOS E DISCUSSÃo}

\section{Bio-ensaios com Anticarsia gemmatalis}

TABELA 1 - Toxicidade de lambda-cyalothrin e monocrotofós para lagartas $\left(3^{\circ}\right.$ instar) de A.gemmatalis por técnica de aplicação tópica.

\begin{tabular}{|c|c|c|c|}
\hline Inseticida & $\begin{array}{l}\text { Avaliação (horas } \\
\text { após a aplicaçãa) }\end{array}$ & $\mathrm{DL}_{30}$ ( $\mu \mathrm{g} /$ inseto) ${ }^{(1)}$ & Equação de regressão \\
\hline $\begin{array}{l}\text { lambda- } \\
\text { cyalothrin }\end{array}$ & $\begin{array}{r}3 \\
6 \\
24 \\
48 \\
72\end{array}$ & $\begin{array}{l}0,0058(0,0046-0,0073) \\
0,0034(0,0020-0,0057) \\
0,0032 \cdot(0,0018-0,0056) \\
0,0023(0,0015-0,0036) \\
0,0017(0,0010-0,0030)\end{array}$ & $\begin{array}{l}y=10,987+2,677 \log x \\
y=9,901+1,982 \log x \\
y=9,872+1,950 \log x \\
y=11,189+2,350 \log x \\
y=11,994+2,532 \log x\end{array}$ \\
\hline monocrotofos & $\begin{array}{c}3 \\
6 \\
24 \\
48 \\
72\end{array}$ & $\begin{array}{cc} & - \\
0,5942 & (0,4279-0,8251) \\
0,3491 & (0,3104-0,3927) \\
0,3075 & (0,2592-0,3649)\end{array}$ & $\begin{array}{c}- \\
y=5,682+3,016 \log x \\
y=6,347+2,947 \log x \\
y=6,643+3,208 \log x\end{array}$ \\
\hline
\end{tabular}

(1) Os valores entre parênteses são os intervalos inferior e superior com $95 \%$ de probabilidade. 
Os dados mostram que $o$ inseticida piretróide lambda-cyalothrin agiu mais rapidamente contra as lagartas de Anticarsia gemmatalis do que o inseticida organofosforado monocrotofós, pois que nas avaliações de 3 e 6 horas o primeiro já havia produzido mortalidades variáveis até $100 \%$, sendo, em conseqüência, possível de serem traçadas as curvas dose $x$ mortalidade $e$ extrapolados os valores de $\mathrm{DL}_{50}$, enquanto que o mesmo não ocorreu com o segundo. Trata-se, este, de um comportamento característico dos inseticidas piretróides em geral, sendo, por isso, os mesmos recomendados em baixas dosagens para uso no campo, inclusive lambda-cyalothrin; isso pode ser vantajoso especialmente em termos do potencial de menor contaminação do meio ambiente.

Os valores de $\mathrm{DL}_{50}$ apresentados na TABELA 1 , bem como os IC $_{95}$, foram transformados na unidade microgramas do inseticida por grama de lagarta $(\mu \mathrm{g} / \mathrm{g})$ (TABELA 2 ).

TABELA 2 - Toxicidade comparativa de lambda-cyalothrin e monocrotofós para lagartas ( $3^{\circ}$ ínstar) de $A$. gemmatalis por técnica de aplicação tópica.

\begin{tabular}{|c|c|c|c|c|c|}
\hline \multirow[t]{2}{*}{$\begin{array}{l}\text { Avaliação } \\
\text { (horas após a aplicação) }\end{array}$} & \multicolumn{2}{|c|}{$\begin{array}{l}\text { lambda-cyalothrin } \\
(\mu \mathrm{g} / \mathrm{g})\end{array}$} & \multicolumn{2}{|c|}{$\begin{array}{l}\text { monocrotofós } \\
(\mu \mathrm{g} / \mathrm{g})\end{array}$} & \multirow{2}{*}{$\begin{array}{l}\text { Toxicidade comparativa } \\
\mathrm{DL}_{50} \text { monocrotof } 6 \mathrm{~s} / \mathrm{DL}_{\mathbf{5}} 0 \\
\text { lambda-cyalothrin }\end{array}$} \\
\hline & DL & $I_{95}$ & $\mathrm{DL}_{50}$ & $I_{86}$ & \\
\hline 3 & 0,09 & $(0,05-0,11)$ & - & - & - \\
\hline 6 & 0,05 & $(0,03-0,09)$ & - & - & - \\
\hline 24 & 0,05 & $(0,02-0,06)$ & 9,29 & $(6,69-12,90)$ & 186 \\
\hline 48 & 0,04 & $(0,02-0,06)$ & 5,46 & $(4,85-6,14)$ & 137 \\
\hline 72 & 0,03 & $(0,02-0,05)$ & 4,81 & $(4,05-5,70)$ & 160 \\
\hline
\end{tabular}

Observa-se que lambda-cyalothrin foi cerca de 140 a 190 vezes mais tóxico do que monocrotofós para lagartas de $3^{\circ}$ ínstar de $A$. gemmatalis (TABELA 2).

\section{Bio-ensaios com Nezara viridula}

TABELA 3 - Toxicidade de lambda-cyalothrin e monocrotofós para ninfas ( $3^{\circ}$ instar) de $N$. viridula por técnica de aplicação tópica.

\begin{tabular}{|c|c|c|c|}
\hline Inseticida & $\begin{array}{c}\text { Avaliação } \\
\text { (horas após a aplicação) }\end{array}$ & $\mathrm{DL}_{s_{0}}(\mu \mathrm{g} / \text { inseto })^{(1)}$ & Equação de regressão \\
\hline \multirow{5}{*}{$\begin{array}{l}\text { lambda- } \\
\text { cyalothrin }\end{array}$} & 3 & $0,0023(0,0016-0,0033)$ & $y=9,539+1,714 \log x$ \\
\hline & 6 & $0,0009(0,0006-0,0013)$ & $y=10,952+1,952 \log x$ \\
\hline & 24 & $0,0007(0,0006-0,0009)$ & $y=10,741+1,828 \log x$ \\
\hline & 48 & - & - \\
\hline & 72 & - & - \\
\hline \multirow{5}{*}{ monocrotofós } & 3 & $0,0951 \quad(0,0491-0,1843)$ & $y=7,550+2,496 \log x$ \\
\hline & 6 & $0,0386(0,0293-0,0510)$ & $y=8,827+2,708 \log x$ \\
\hline & 24 & $0,0143(0,0088-0,0234)$ & $y=8,825+2,106 \log x$ \\
\hline & 48 & + & - \\
\hline & 72 & - & - \\
\hline
\end{tabular}

(1) Os valores entre parênteses são os intervalos inferior e superior com $95 \%$ de probabilidade. 
Não foram calculados os parâmetros correspondentes a 48 e 72 horas após a aplicação em razão das altas porcentagens de mortalidade nas parcelas testemunhas (média acima de $20 \%$ ).
Os valores $\mathrm{DL}_{50}$ apresentados na TABELA 3, bem como os IC 95 , foram transformados na unidade microgramas do inseticida por grama de ninfa ( $\mu \mathrm{g} / \mathrm{g})$ (TABELA 4).

TABELA 4 - Toxicidade comparativa de lambda-cyalothrin e monocrotofós para ninfas $\left(3^{\circ}\right.$ ínstar) de $N$. viridula por técnica de aplicação tópica.

\begin{tabular}{|c|c|c|c|c|c|}
\hline \multirow[t]{2}{*}{$\begin{array}{c}\text { Avaliação } \\
\text { (horas ap6s a aplicaçăo) }\end{array}$} & \multicolumn{2}{|c|}{$\begin{array}{l}\text { lambda-cyalothrin } \\
(\mu \mathrm{g} / \mathrm{g})\end{array}$} & \multicolumn{2}{|c|}{$\begin{array}{l}\text { monocrotofos } \\
(\mu \mathrm{g} / \mathrm{g})\end{array}$} & \multirow{2}{*}{$\begin{array}{l}\text { Toxicidade } \\
\text { comparativa } \\
\text { DL } \\
\text { monocrotofós/DL } \\
\text { lambda-cyalothrin }\end{array}$} \\
\hline & DL & $\mathrm{IC}_{95}$ & $\mathrm{DL}_{\mathbf{3 0}}$ & IC $_{95}$ & \\
\hline 3 & 0,82 & $(0,57-1,18)$ & 33,96 & $(17,54-65,82)$ & 41 \\
\hline 6 & 0,32 & $(0,21-0,46)$ & 13,79 & $(10,46-18,21)$ & 43 \\
\hline 24 & 0,25 & $(0,21-0,32)$ & 5,11 & $(3,14-8,36)$ & 20 \\
\hline 48 & - & - & - & - & - \\
\hline 72 & - & - & - & - & - \\
\hline
\end{tabular}

Observa-se que lambda-cyalothrin foi cerca de 20 a 40 vezes mais tóxico do que monocrotofós para ninfas de $3^{\circ}$ ínstar de $N$. viridula (TABELA 4).
Com os dados das TABELAS 2 e 4 foi elaborada a TABELA 5, que expressa a toxicidade comparativa de lambda-cyalothrin e monocrotofós para os insetos estudados.

TABELA 5 - Toxicidade comparativa de lambda-cyalothrin e monocrotofós para lagartas ( $3^{\circ}$ ínstar) de $A$. gemmatallis e a ninfas $\left(3^{\circ}\right.$ ínstar) de $N$. viridula.

\begin{tabular}{|c|c|c|c|c|c|c|}
\hline \multirow{2}{*}{$\begin{array}{l}\text { Avaliação } \\
\text { (horas ap6́s } \\
\text { a aplicação) }\end{array}$} & \multicolumn{3}{|c|}{ lambda-cyalothrin } & \multicolumn{3}{|c|}{ monocrotofos } \\
\hline & $\begin{array}{c}\mathrm{DL}_{50} \\
\text { (ug/g) } \\
\text { A.gemmatalis }\end{array}$ & $\begin{array}{c}\mathrm{DL}_{\mathbf{s p}_{0}} \\
\text { (ug/g) } \\
\text { N.viridula }\end{array}$ & $\begin{array}{c}\text { Toxicidade } \\
\text { comparativa }^{(1)}\end{array}$ & $\begin{array}{c}\mathrm{DL}_{5_{0}} \\
(\mu \mathrm{g} / \mathrm{g}) \\
\text { A.gemmatalis }\end{array}$ & $\begin{array}{c}\mathbf{D L}_{50} \\
\text { (ug/g) } \\
\text { N.viridula }\end{array}$ & $\begin{array}{c}\text { Toxicidade } \\
\text { comparativa }^{(2)}\end{array}$ \\
\hline 3 & 0,09 & 0,82 & 9 & - & 33,96 & - \\
\hline 6 & 0,05 & 0,32 & 6 & - & 13,79 & - \\
\hline 24 & 0,05 & 0,25 & 5 & 9,29 & 5,11 & 2 \\
\hline 48 & 0,04 & - & - & 5,46 & - & - \\
\hline 72 & 0,03 & - & - & 4,81 & - & - \\
\hline
\end{tabular}

(i) $\mathrm{DL}_{50} N$. viridula/DL $\mathrm{DL}_{50}$ A. gemmatalis

(2) $\mathrm{DL}_{50}$ A. gemmatalis/DL $\mathrm{L}_{50} N$. viridula 
Observa-se que lambda-cyalothrin foi cerca de 5 a 9 vezes mais tóxico às lagartas de $A$. gemmatalis do que às ninfas de $N$. viridula, e que monocrotofós, ao contrário, foi cerca de duas vezes mais tóxico às ninfas.

\section{CONCLUSÕES}

- Lambda-cyalothrin agiu mais rapidamente contra lagartas de $3^{\circ}$ ínstar de Anticarsia gemmatalis do que monocrotofós;

- A toxicidade de lambda-cyalothrin foi cerca de 140 a 190 vezes maior do que a de monocrotofós para estes insetos;

- A toxicidade de lambda-cyalothrin foi, também, maior do que a de monocrotofós, cerca de 20 a 40 vezes, para ninfas de $3^{\circ}$ ínstar de $N$. viridula;

- Lambda-cyalothrin foi cerca de 5 a 9 vezes mais tóxico para $A$. gemmatalis do que a $N$. viridula;

- Monocrotofós foi cerca de duas vezes mais tóxico para $N$. viridula do que $A$. gemmatalis.

\section{REFERÊNCIAS BIBLIOGRÁFICAS}

CORRÊA-FERREIRA, B.S. Criação massal do percevejo verde Nezara viridula (L.). Londrina: EMBRAPA/CNPSo, 1985. 16p. (Documentos, 11).

ELZEN, G.W.; O'BRIEN, P.J.; SNODGRASS, G.L. Toxicity of various classes of insecticides to pyrethroid resistant Heliothis virescens larvae. Southwestern Entomologist, Dallas, v.15, p.33-38, 1990.

GABRIEL, D.; CALCAGNOLO, G.; TANCINI, R. S.; GONÇALVES, P.C.T.; SIQUEIRA, D.F. Controle químico do bicudo-do-algodoeiro,Anthonomus grandis Boheman, 1843 (Coleoptera; Curculionidae) através de pulverização eletrodinâmica, em condiçōes de campo. Anais da Sociedade Entomologica do Brasil, Porto Alegre, v.19, p.87-93, 1990.

GALLO, D.; NAKANO, O.; SILVEIRA NETO, S.; CARVALHO, R.P.L.; BATISTA, G.C. de; BERTI FILHO, E.; PARRA, J.R.P.; ZUCCHI, R.A.; ALVES, S.B.; VENDRAMM, J.D. Manual de entomologia agnícola. 2.ed. São Paulo: Agronômica Ceres, 1988. 649 p.

GREENE, G.L.; LEPPLA, N.C.; DICKERSON, W.A. Velvetbean caterpillar: a rearing procedure and artificial medium. Journal of Economic Entomology, Laham, v 69, n.4, p.487-488, 1976.
LEONARD, B.R.; SPARKS, T.C.; GRAVES, J.B. Insecticide cross-resistance in pyrethroid-resistant strains of tobacco budworm (Lepidoptera: Noctuidae). Journal of Economic Entomology, Lanham, v.81, n.1, p.1529-1535, 1988.

LEONARD, B.R.; BOETHEL, D.J.; SPARKS JUNIOR, A.N.; LAYTON, M.B.; MINK, J.S.; PAVLOFF, A.M.; BURRIS, E.; GRAVES, J.B. Variations in response of soybean looper (Lepidoptera; Noctuidae) to selected insecticides in Louisiana. Journal of Economic Entomology, Lanham, v.83, n.1, p.27-34, 1990.

MARTINS, J.C.; VALERIO, M.A.; MOREIRA, L.A. Formulações ED no controle do percevejo verde Nezara viridula (L., 1758) (Hemiptera: Pentatomidae) na cultura da soja. Anais da Sociedade Entomologica do Brasil, Porto Alegre, v.19, n.1, p.51-58, 1990.

MARTINS, J.C.; VALÉRIO, M.A.; MOREIRA, L.A.; BERTUCCI, P.C.D. Formulaçōes ED no controle da lagarta rosada, Pectinophora gossypiella (Saunders, 1844) (Lepidoptera: Gelechiidae) na cultura do algodão. Anais da Sociedade Entomológica do Brasil, Porto Alegre, v.17, n.2, p.389-396, 1988.

N'GUESSAN, K.F.; CHALFANT, R.B. Dose response of the cowpea curculio (Coleoptera: Curculionidae) from different regions of Georgia to some currently used pyrethroid insecticides. Journal of Entomological Science, Tifton. v.25, p.219-222, 1990.

PARRA, J.R.P. Criação de insetos para estudos com patógenos. In: ALVES, S.B. (Coord.). Controle Microbiano de Insetos. São Paulo: Manole, 1986. p.348-373.

SAXENA, J.D.; SRIVASTAVA, K.M.; RAI, S.; SINHA, S.R.; PAUL, A.V.N. Base-line toxicity of some insecticides to a laboratory susceptible strain of Heliothis armigera Hubner. Indian Joumal of Entomology, New Delhi, 51(3):352-354, 1989. Apud Review of Agricultural Entomology, Wallingford, 78(12):1385, 1990. (Resumo).

SU, N.Y.; SCHEFFRAHN, R.H. Comparison of eleven soil termiticides against the Formosan subterranean termite and eastern subterranean termite (Isoptera: Rhinotermitidae). Joumal of Economic Entomology, Lanham, v.83, n.5, p.1918-1924, 1990.

Trabalho entregue para publicação em 30.06 .94

Trabalho aprovado para publicação em 25.10 .94 